\title{
Optical enhancement by means of concentration tuning of gold precursors in polymer nanocomposite materials
}

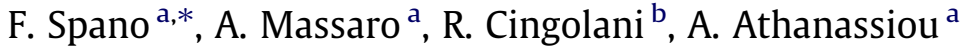 \\ a Italian Institute of Technology IIT, Center of Bio-Molecular Nanotechnologies, Arnesano 73100, Italy \\ ${ }^{\mathrm{b}}$ Italian Institute of Technology IIT, Via Morego 30, 16163 Genova, Italy
}

\section{A R T I C L E I N F O}

Article history:

Available online 25 January 2011

\section{Keywords:}

Gold precursor

Polymer film

Nanocomposite material

Optical property tuning

Optical sensor

\begin{abstract}
A B S T R A C T
In this work, we propose a new approach regarding the characterization and the enhancement of transmitted light travelling inside a chitosan polymeric film improved by implementation of gold precursors generating nanocomposite materials. Firstly, the experimental optical characterization indicates the influence of the presence of gold particles on the effective refractive index of the chitosan polymer films and, secondly, the influence of their thickness supported by the transmittivity enhancement at precise frequencies for each gold precursor concentration and each polymer film thickness. This developed analysis introduces a basic study for the light enhancement in future complex photonic devices suitable for sensing.
\end{abstract}

(c) 2011 Elsevier B.V. All rights reserved.

\section{Introduction}

Nanocomposite research is constantly exploring and developing new applications. In this direction, we focus on the combination of polymers and filler components creating nanocomposite materials for optical applications. Such combination allows us the preparation of tailored materials dedicated to specific applications. The need for novel optically functional and transparent materials is increasing due to the expansion of applied optics. In addition to optical needs such as high sensitivity and detection enhancement, in many cases materials need to be integrated in systems including waveguides and optical fibers. Therefore, films and fibers are of great interest as the final form of these novel materials [1-5]. The reason for the use of nanocomposite materials in this work is that they combine the flexibility and easy processability of the polymers with the optical properties of the fillers used. In particular we introduce the use of nanocomposite materials for future robots applications where the optical enhancement is requested. Our strategy is based on the basic use of gold precursors as chloroauric acid salt introduced in transparent polymer films. In that precise case, chitosan (CTO) polymer was chosen for its biocompatibility and also for future biosensing applications but even for its ability to generate gold nanoparticles in particular conditions [6-8], particularity developed and characterized in further studies elsewhere.

\footnotetext{
* Corresponding author.

E-mail addresses: fabrizio.spano@iit.it (F. Spano), alessandro.massaro@iit.it (A Massaro).
}

This preliminary work represents the first approach to the design of optical resonators based on gold nanocomposite materials. Through this article is illustrated the influence of gold precursor concentration and of the polymer film thickness on the increase of transmitted light through the nanocomposite film, and consecutively on the nanocomposite efficiency for optical detection. This study is the basic step for a design of an optical waveguide detector. We found that the increase of the gold precursor concentration leads to an increase of the transmitted light intensity. The effective refractive index of the gold nanocomposite material waveguide can be approximately estimated assuming spherical gold particles in CTO polymeric film. Theoretical calculations show a strong variation of the CTO refractive index due to the gold precursor concentration [9], that is in agreement with our experimental findings for improved optical sensing due to gold precursor, measured by enhanced transmittivity. We observed that for higher film thickness the transmittivity of the film increases at specific wavelengths.

\section{Fabrication process of CTO polymer film and experimental optical characterizations}

A characteristic thin layer film of CTO polymer on glass substrate in which is introduced gold precursor particles is shown in Fig. 1a. The polymeric film is obtained mixing chitosan polymer (Sigma Aldrich - 448877) with acetic acid (1\% - Sigma Aldrich $242853)$. Various concentrations of CTO polymer solutions $(0.5 \%$, $1 \%$, and $2 \%$ in weight) are prepared. CTO polymeric films are obtained by drop-casting $\left(50 \mu \mathrm{L} / \mathrm{cm}^{2}\right)$. The gold precursor used is a chloroauric acid salt (Sigma Aldrich - 254169, $\mathrm{Mw}\left(\mathrm{HAuCl}_{4}\right)=$ $339.5 \mathrm{~g} / \mathrm{mol}$ ) and is dissolved in distilled water by providing two 


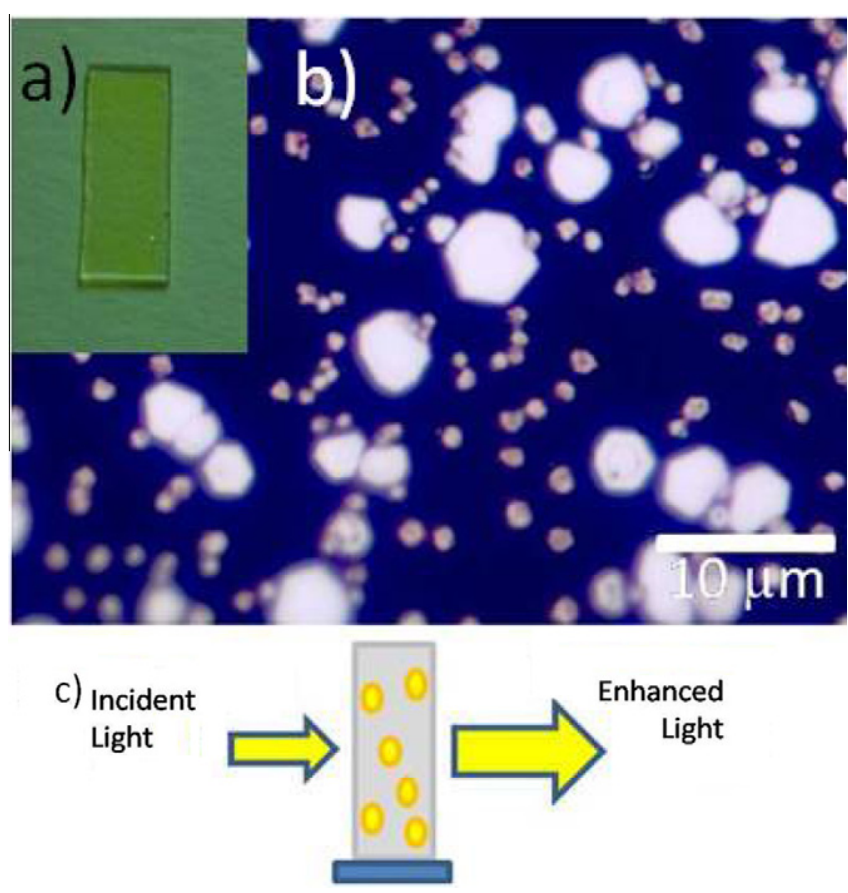

Fig. 1. (a) Photo of the CTO polymer film in which is introduced gold precursor particles. (b) Optical high magnification image of gold precursors crystals embedded in CTO polymer film. (c) Schematic illustration of the light enhancement expected by introduction of gold precursors crystals.

solutions $\left(\left[\mathrm{HAuCl}_{4}\right]=0.01 \mathrm{M}\right.$ and $\left.0.02 \mathrm{M}\right)$. During the preparation of the gold nanocomposite film, the gold precursor is introduced in the drop-casted polymeric film by immersion process. To realize this experiment, CTO films are directly immersed into gold precursor solutions during $1 \mathrm{~h}$. The introduction of gold precursor by immersion allows us to control and avoid the generation of gold nanoparticles into the CTO polymeric film. An external stimulation is needed to start the nanoparticle generation process. CTO polymer is known to be a good stabilizing/reducing agent of gold precursor $[10,11]$. Nevertheless, this particularity will be developed for specific applications in our next works focused on generation of gold nanocomposite materials. To illustrate the aspect of the nanocomposite material, an optical high magnification $(100 \times)$ image indicates the presence of gold precursor crystals embedded into CTO polymer film in Fig. 1b. In Fig. 1c, we illustrate the expected mechanism due to the change of effective refractive index obtained by the addition of gold precursor particles into the nanocomposite material leading to the transmittivity amplification. Fig. 2a illustrates a modelling of gold particles introduced into the CTO polymeric film allowing us to consider the nanomaterial as a thin metallic layer: the high concentration allows assuming the gold particles as resonators because their distance is of order of the working wavelength. We experimentally characterize our CTO polymer film by using a broad band lamp source (Fig. 2b): the electromagnetic field is transmitted through the sample vertically fixed illustrated by the photo in Fig. 2c and the signal is measured by an optical multichannel analyzer (OMA). As illustrated by Fig. 3, immersion times allows to control or tune the gold precursor concentration introduced into the CTO polymeric film. Moreover, as observed in Fig. 3a, FTIR measurements made on a $0.5 \%$ CTO polymer film indicate the chemical modification of the CTO polymeric film due to the absorption of gold precursor particles by means of light intensity enhancement. The comparison between the 20 min immersion curve (black) with the two 40 min (red) and $60 \mathrm{~min}$ (blue) immersion curves indicates the kinetic of gold

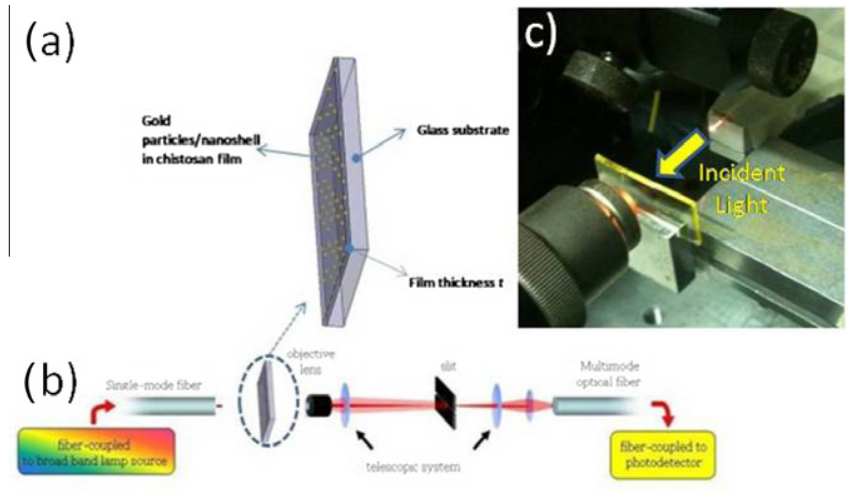

Fig. 2. (a) Modelization of the CTO polymer film with gold precursor particles. (b) Schematic configuration of the measurement dispositive by means of an optical multichannel analyzer and a broad lamp source. (c) Photo illustrating the vertical positioning of the sample during measurement using incident light beam.

precursor absorption in CTO polymeric films. The superposition of the 40 and 60 min immersion curves indicate the existence of an absorption maximum of gold precursor in CTO films for a precise CTO polymer concentration by light intensity measurements. By changing the CTO polymer concentration as illustrated on Fig. 3b, curves revealed an increase of the light intensity illustrating the influence of the CTO polymer concentration where $1 \%$ CTO film seems to absorb more gold precursor than the $0.5 \%$ CTO film. Immersion times allow to tune the gold precursor concentration introduced into the CTO polymeric film by reaching a gold saturation when the immersion time is of about 40 min for a CTO concentration of $1 \%$ (about $2 \mu \mathrm{m}$ of thickness). The saturation time is also function of the film thickness and by the way from CTO polymer concentration. We have observed during our experimentation that increasing the CTO concentration we observe an increase of the film thickness. Moreover, we observe that the CTO polymer behaves as a hydrogel. This property is used to tune the gold concentration in the CTO polymer film.

The observation must be correlated to the Fig. 4 where are represented the variation of CTO polymeric film thicknesses in function of the CTO polymer concentration. The experiment results indicate that the increase of CTO polymer concentration increase the CTO film thickness. This result may explain the variation of the previous FTIR curves seen earlier where the 1\% CTO film (Fig. 3b) generates an higher light intensity enhancement than the $0.5 \%$ CTO film (Fig. 3a). After the introduction of the hypothesis linking the variation of CTO film thickness with the ability of the CTO film to absorb a higher amount of gold precursor, the observation of the Fig. 5 may help us to understand the influence of this increase of gold concentration on optical transmittivity measurements. Fig. 5a and b illustrate respectively the UV-vis and the FTIR spectra obtained for CTO polymer film where CTO polymer and gold precursor concentrations are changed. We conclude that the light enhancement can be improved or by a long time of immersion process, by fixing the gold precursor concentration, or, by increasing the gold precursor concentration correlated with the CTO polymer concentration (more high is the CTO concentration, more gold precursor can be implemented inside the polymer film). Observed trends indicate the increase of transmitted light intensity due to the correlated increase of the CTO polymer and gold precursor concentrations. Observations confirmed by Fig. 6a and b where are illustrated, respectively, the experimental measurements of light intensity indicating the influence of the gold particles on the effective refractive index of the CTO polymer films and the influence of their thickness supported by the transmittivity enhancement at precise frequencies for each gold precursor concentration and each 
(a)

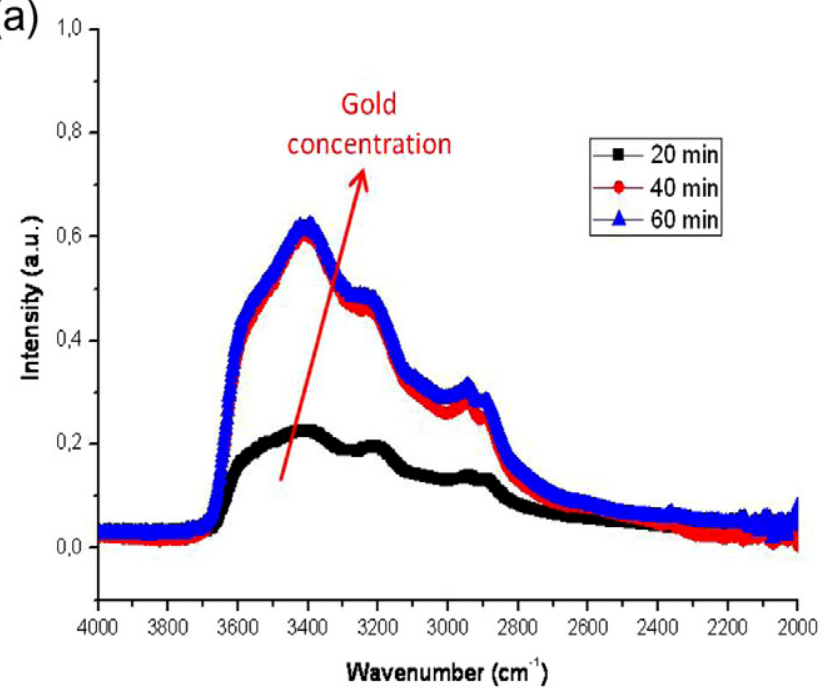

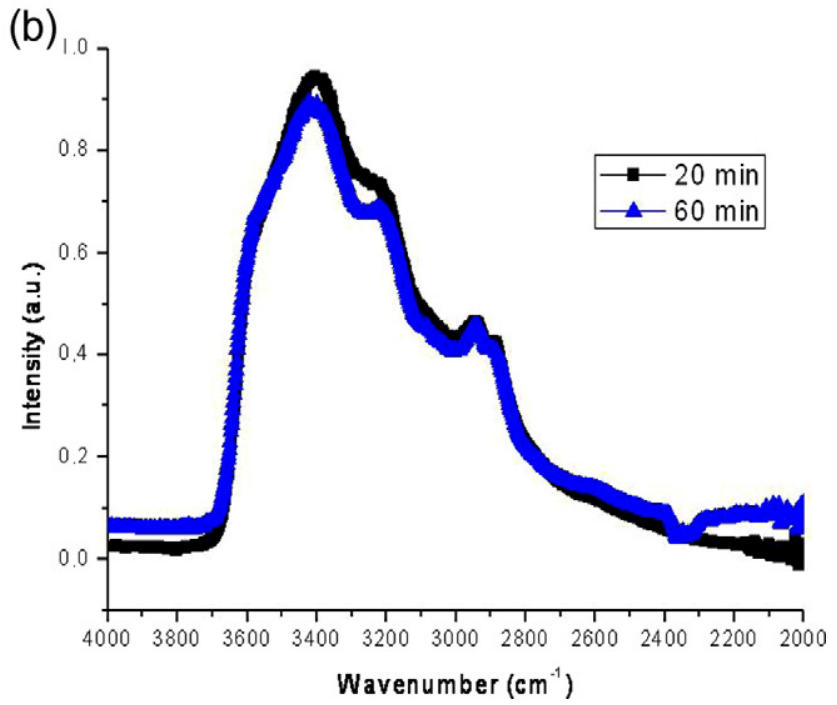

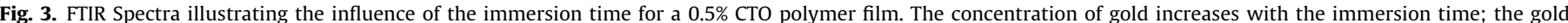

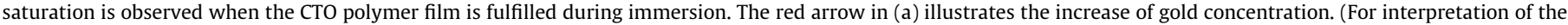
references to colour in this figure legend, the reader is referred to the web version of this article.)

(a)

\begin{tabular}{|c|c|c|}
\hline $\begin{array}{c}\text { Chitosan Polymer } \\
\text { Concentration }(\% \mathrm{w} / \mathrm{w})\end{array}$ & $\begin{array}{c}\text { Chitosan Polymer } \\
\text { Thickness }(\mu \mathrm{m})\end{array}$ & $\begin{array}{c}\text { Layer } \\
\text { number }\end{array}$ \\
\hline 0,5 & 1.5 & 1 \\
1 & 1.8 & 1 \\
1 & 3.2 & 2 \\
2 & 3.5 & 1 \\
\hline
\end{tabular}

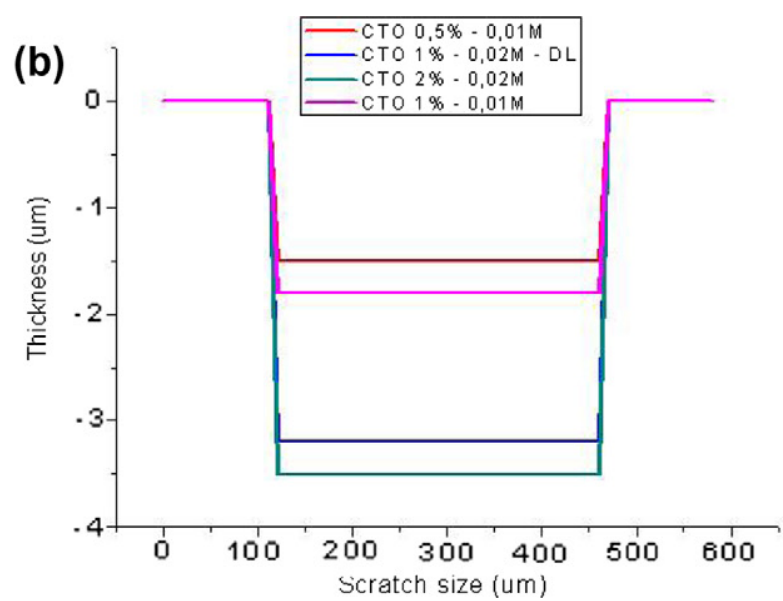

Fig. 4. (a) Table indicating by experimental measurements the thickness of a СTO polymer film made by contact profilometry for various CTO concentrations. (b) Curves indicating by experimental measurements the thickness of a CTO polymer film made by contact profilometry for various CTO concentrations.

(a)

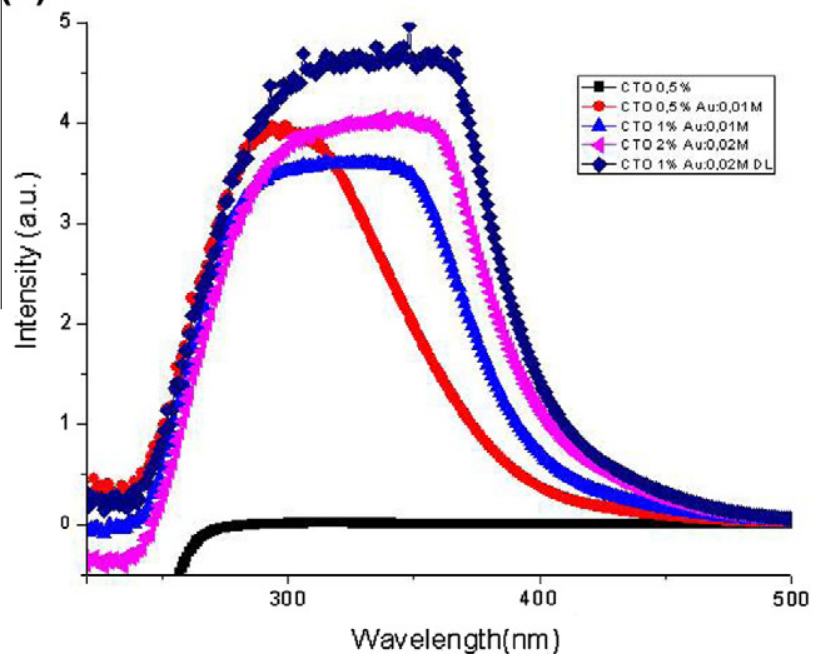

(b)

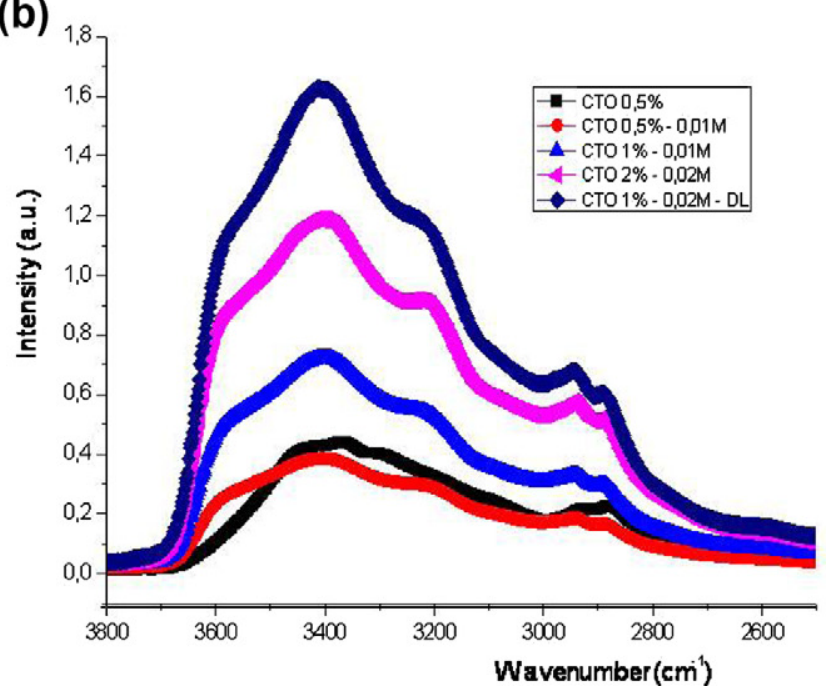

Fig. 5. (a) UV-vis spectra of CTO polymeric films with various gold precursor and CTO polymer concentrations. (b) FTIR spectra of CTO polymeric films with various gold precursor and CTO polymer concentrations. 

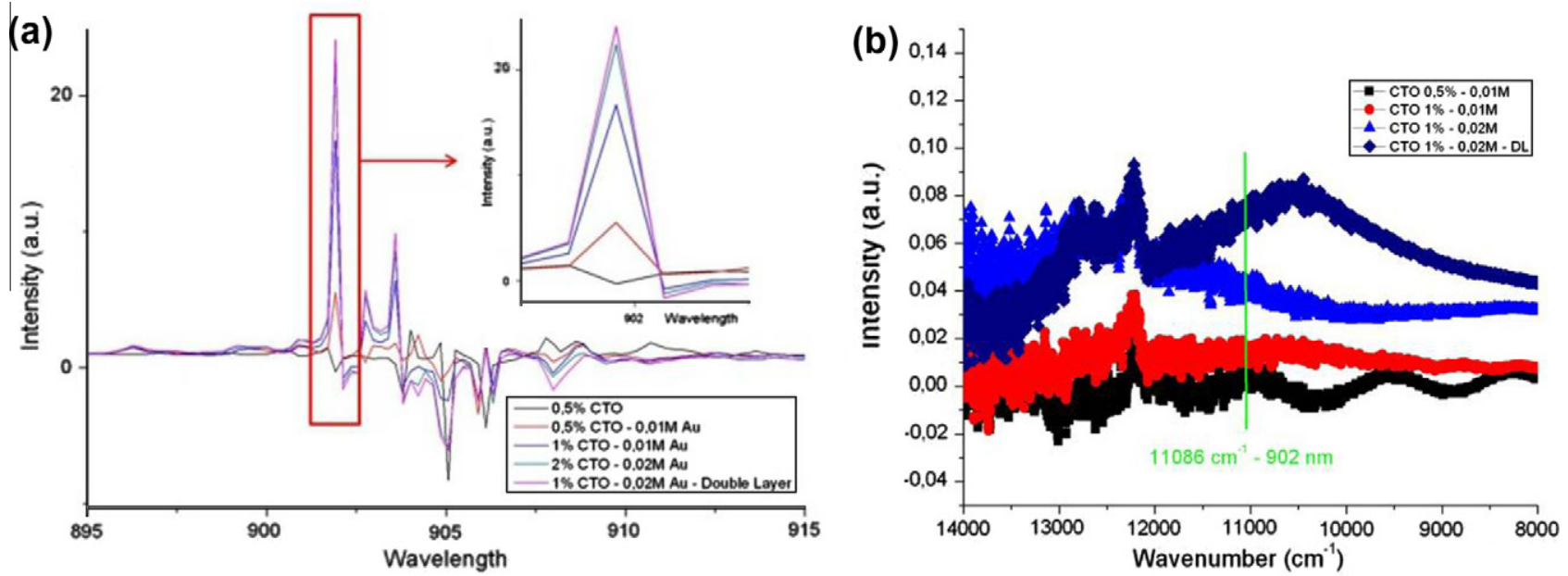

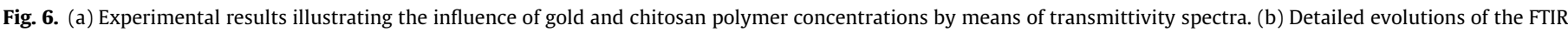
curves at precise wavenumbers illustrating the same trend observed in transmittivity measurements.

polymer film thickness. Moreover, we observe from Fig. $6 \mathrm{~b}$ that the absorption effect is more evident starting from 11,500 to $8500 \mathrm{~cm}^{-1}$.

\section{Conclusion}

We present in this work a preliminary study about optical enhancement of polymeric nanocomposite film using gold precursors. The analysis of the optical measurements of nanocomposite mats indicate two principle influences: the influence of the gold precursor concentration and in the same manner the influence of the CTO polymer concentration. The experimental results indicate an increase of the CTO polymeric thickness with the CTO concentration leading to the increase of the polymeric film capacity to absorb gold precursors. These two observations explain the transmittivity enhancement observed in our preliminary results. We proved that a light enhancement can be performed by increasing the Au concentration or by increasing the CTO concentration or by immerging at least for $40 \mathrm{~min}$ the CTO polymer film into gold precursor solution. The study will be extended to the influence of the size of the gold particles from micro to nano particles. The approach will be applied for the interpretation of future experimentations concerning the evanescent field generated by gold nanocomposite films for electromagnetic field enhancement and directly used in technological devices for optical sensing. The study of the gold particles size is actually under investigation.

\section{References}

[1] G.J. Hutchings, M. Brust, H. Schmidbaur, Chem. Soc. Rev. 37 (2008) 1759-1765

[2] M. Brust, D. Bethell, C.J. Kiely, D.J. Schiffrin, Langmuir 14 (1998) 5425-5429.

[3] M.A. Martins, S. Fateixa, A.V. Girao, S.S. Pereira, T. Trindade, Langmuir 26 (2010) 11407-11412.

[4] H. Du, J.Q. Xiao, Y.S. Zou, T.G. Wang, J. Gong, C. Sun, L.S. Wen, Opt. Mater. 28 (2006) 944-949.

[5] L.L. Beecroft, C.K. Ober, Chem. Mater. 9 (1997) 1302-1317.

[6] V. Myroshnychenko, J. Rodriguez-Fernandez, I. Pastoriza-Santos, A.M. Funston, C. Novo, P. Mulvaney, L.M. Liz-Marzan, F.J. Garcia de Abajo, Chem. Soc. Rev. 37 (2008) 1792-1805.

[7] M.K. Abyaneh, D. Paramanik, S. Varma, J. Phys. D Appl. Phys. 40 (2007) 37713779.

[8] A. Goyal, A. Kumar, P.K. Patra, S. Tabatabaei, P.J.J. Alvarez, G. John, P.M. Ajayan, Macromol. Rapid Commun. 30 (2009) 1116-1122.

[9] M. Chen, R.G. Horn, J. Colloid Interface Sci. 315 (2007) 814-817.

[10] T. Miyama, Y. Yonezawa, Langmuir 20 (2004) 5918-5923.

[11] H. Huang, Q. Yuan, X. Yang, J. Colloid Interface Sci. 282 (2005) 26-31. 